\title{
Smart Kote Glove for Assessment of Scoring Parameters of Dan and Kyu Grade Kendokas ${ }^{\dagger}$
}

\author{
Kwangyul Jeong *, Adin Ming Tan and Franz Konstantin Fuss \\ Smart Equipment Engineering and Wearable Technologies Research Program, Centre for Design Innovation, \\ Swinburne University of Technology, Melbourne, VIC 3122, Australia; kjeong@swin.edu.au (K.J.) \\ amtan@swin.edu.au (A.M.T.); fkfuss@swin.edu.au (F.K.F.) \\ * Correspondence: kjeong@swin.edu.au; Tel.: +61-03-3921-45320 \\ + Presented at the 12th Conference of the International Sports Engineering Association, Brisbane, \\ Queensland, Australia, 26-29 March 2018.
}

Published: 13 February 2018

\begin{abstract}
Kendo is one of the most ancient swordsmanship arts in Japan. The aims of this study are to develop and test an innovative smart Kote glove for assisting the Kendoka to distinguish scoring from non-scoring Kote (wrist) strikes. An in-house developed pressure sensing platform was utilized to develop the smart Kote glove. Ten kendo practitioners, comprising of five Dan (black belts equivalent) and five Kyu (lower level/ungraded of both genders), participated in this study. The results showed significant differences between Dan and Kyu participants in both accuracy and sharpness of the strikes. Dan grade participants showed higher percentage of hitting the target comparing to Kyu grade ( $92 \%$ and $75 \%$ respectively). The percentage of scoring was also significantly higher in Dan (78\%) than in Kyu (37\%) grades. The average impact force of scoring by Dan grade $(1159 \pm 379 \mathrm{~N})$ was higher than by Kyu grade $(852 \pm 429 \mathrm{~N})$.
\end{abstract}

Keywords: kendo; smart kote glove; scoring sensor; automatic scoring system; pressure sensing platform; sport technology

\section{Introduction}

Sensor technology has been utilised in automatic scoring during the past decade in many sports such as martial arts [1] and winter sports [2]. The technology was introduced to aid human umpire in judging the sport game accurately and consistently, especially in the large sports competitions such as Olympic.

In terms of scoring sensors used in martial arts, there are several Olympic martial arts currently utilising automatic scoring sensors as part of the judging criteria. In fencing, electronic scoring was first introduced to Olympic fencing for épée in 1936, foil in 1956, and sabre in 1988. The central unit of the scoring system is commonly known as "the box". In the simplest version, both fencers' weapons are connected to the box via long retractable cables. The box normally carries a set of lights to signal when a touch has been made [3]. In Taekwondo, electronic protector judgement system has been first adopted in 2007 and later implemented in the 2012 London Olympic. Impact force sensors have been installed onto body protectors and head gears (Hogu) [4]. However, to date, there is no electronic scoring system used in Kendo.

There are few studies carried out on sensor technology in Japanese Swordsmanship so far. Sensors have been installed directly onto martial arts equipment for training purposes. James et al. have incorporated accelerometers to a wooden sword to study the dynamics of swings [5]. Another study carried out by Jeong et al. [6] was utilising pressure sensors attached to a bamboo sword (Shinai), used in Kendo, to measure grip pressure. The study focused on the pressure distribution 
between left and right hand during normal stance and an attacking motion to assist beginners in cultivating correct hand placements in Kendo.

"The way of Sword" is the Japanese meaning of Kendo which is one of the most ancient martial arts [7]. There are four components of the Kendo armour: Men, Kote, Do and Tare [8] (see Figure 1). Men is a helmet with metal grill to protect the head and face area and a series of hard leather and fabric flaps to protect the throat, the side of the neck and shoulders. Kote is a thickly padded fabric glove used to protect the forearms, wrists and hands. The torso is protected by a breastplate called Do, while the waist and groin area is protected by a thick padded fabric flaps called Tare [8]. Kendo training requires several years to achieve high standard level, which is called 'Dan'. The novice Kendoka(s) usually start as 'Kyu' graders and work their way up the ladder to 8th Dan. It normally takes over 30 years of active training to move up from 1st Dan to 8th Dan [9]. All Kendokas usually train repetitive motions to perfectly match their physical and mental states in Kendo under the concept of Ki (spirit)-Ken (Sword)-Tai (Body). This requires many years of guided training by Kendo instructors (Sensei) and seniors.

In order to score, a strike by a bamboo sword has to be placed in the correct area of the targets (Men/head, Kote/wrist, Do/torso and Tsuki/throat) with accuracy and sufficient force [7]. Therefore, it is essential for both players and the Shinpans (umpires) to be able to recognize these parameters for assessing the scoring motion. The aims of this study are to develop and test an innovative smart Kote glove for assisting the Kendoka to distinguish scoring from non-scoring strikes. This will help facilitate learning of kendo techniques with improvement of performance in competitions.

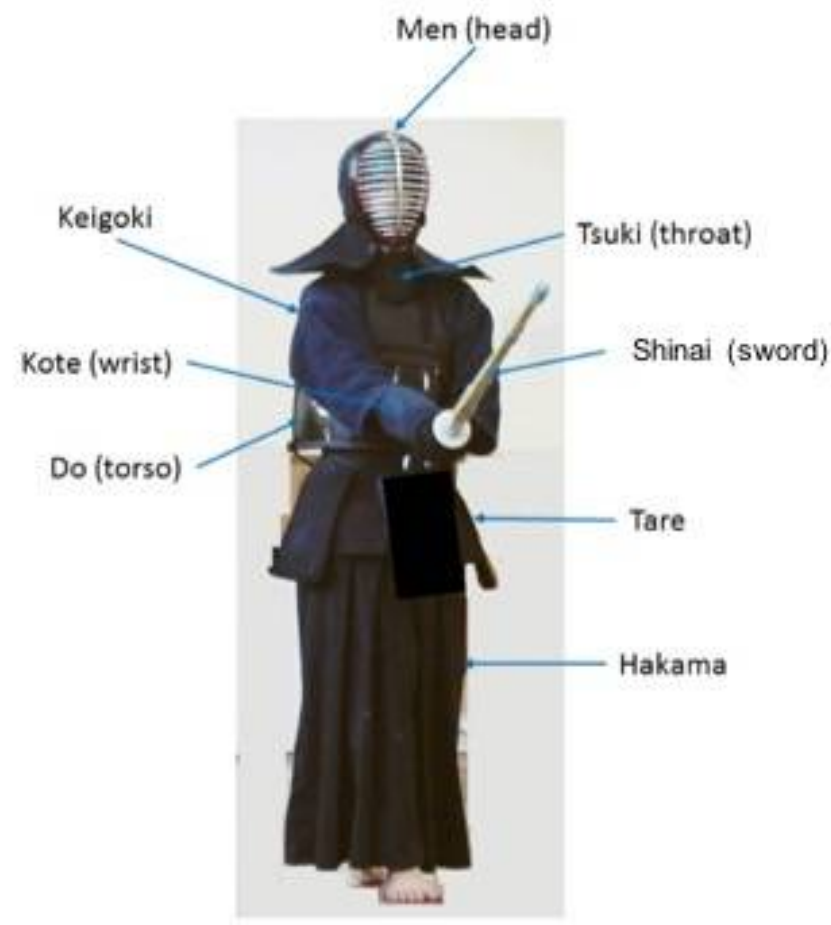

Figure 1. Illustration of kendo uniform (Keigoki and Hakama), armours (Men, Kote, Do and Tare) and sword (Shinai); Photo courtesy@ University of Melbourne Kendo club, reproduced with kind permission.

\section{Design and Method}

\subsection{Design Rationale}

The wrist target (Kote) is considered to be the most difficult to judge by the umpire (Shinpan). This is because the target is relatively small and not perfectly visible as compared to other targets. 
Therefore, a Kote scoring system was designed by utilizing a pressure sensor and a data acquisition system (USB-2404-10, Measurement Computing, Norton, MA, USA) (Figure 2).

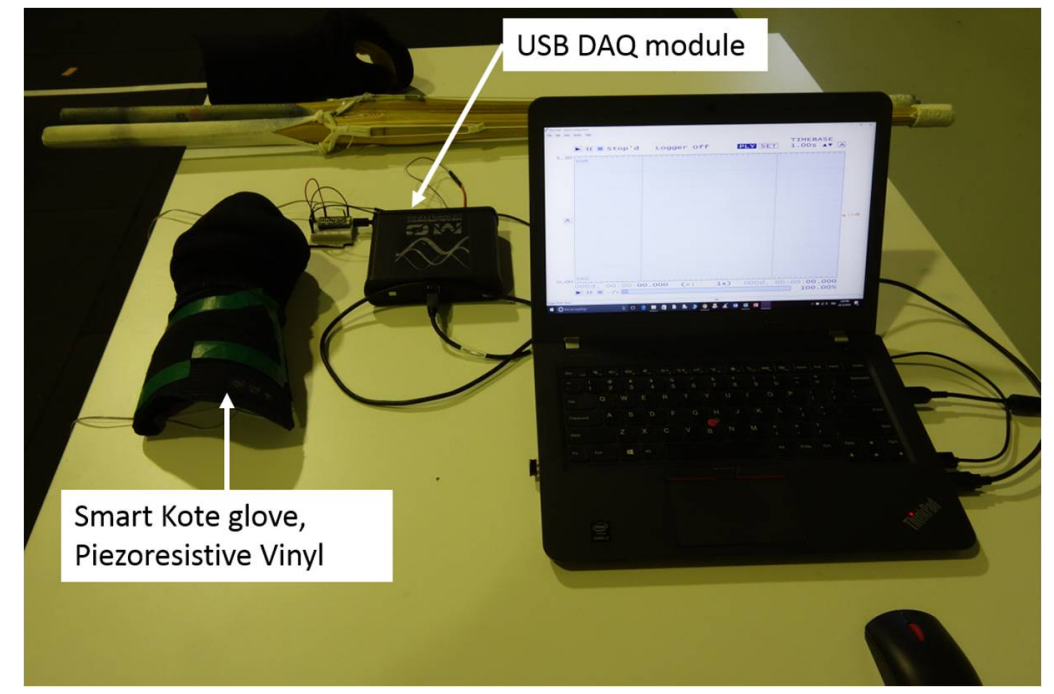

Figure 2. Experiment set up: in-house developed pressure sensor for Kote glove and DAQ module.

\subsection{Electronics}

Instead of using FSR, FlexiForce or similar off-the-shelf sensors for pressure measurements, an in-house developed pressure sensing platform was utilized to develop the smart Kote glove. The lowcost but very accurate sensors were made of piezo-resistive vinyl with conductive fabric (Ripstop, DEV-10056) used as electrodes. The electrodes were taped to the glove's polymer structure and connected with wires to a potential divider circuit and finally to a four channel USB DAQ module (USB-2404-10, Measurement Computing, Norton, MA, USA). A power supply of $3.3 \mathrm{~V}$ was used to drive the circuit. TracerDAQ ${ }^{\circledR}$ Pro (Measurement Computing, Norton, MA, USA) was used for data recording at $10 \mathrm{kHz}$.

\subsection{Experimental Procedure}

Ten kendo practitioners, comprising of five Dan Kendokas (black belts equivalent) and five Kyu (lower level/ungraded athletes of both genders), participated in this study. The Dan Kendokas were requested to execute a Debana Kote (a counter attack technique of striking the wrist target while the opponent was striking the head target) for a total of 15 times on an opponent wearing the smart Kote glove (Figure 3). On the other hand, the Kyu Kendokas were requested to execute the Debana Kote for a total of 20 times. The study was approved by the Ethics Committee (SUHREC) of Swinburne University of Technology (2016/296).

\subsection{Data Processing}

The voltage drop across the sensor was recorded and the conductivity of the sensor in milliSiemens (mS) was calculated. Using the data from our previous study[10], the conductance was converted to impact force, and ultimately to four scoring parameters: score (above an impact conductance of $20.2 \mathrm{mS}$ ), no score (below $17 \mathrm{mS}$ ), grey-zone (between the two thresholds) and missing the target (impact outside the sensor area or no impact spike recorded). 


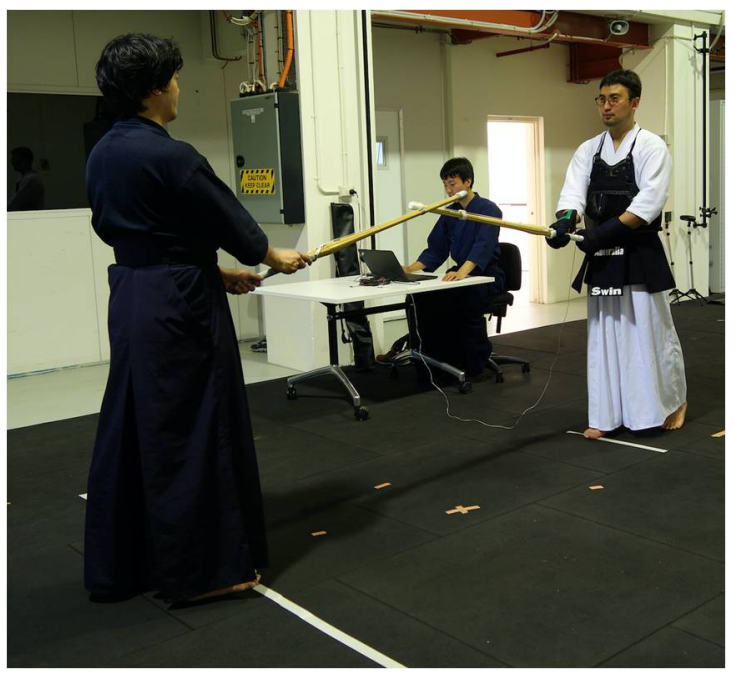

(a)

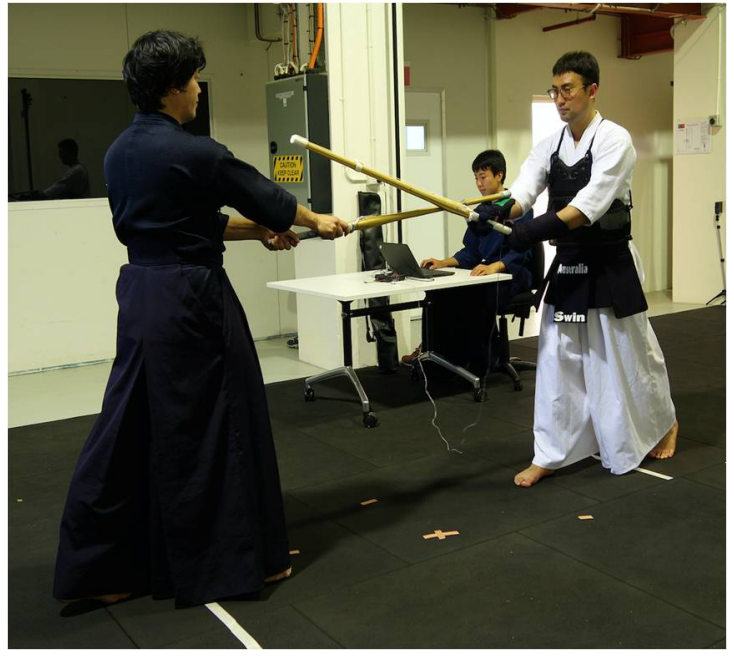

(b)

Figure 3. (a) Kendoka in experimental position for testing the scoring parameters; (b) Posture when performing Debana Kote (striking the wrist).

\section{Results}

The results showed significant differences between Dan and Kyu participants in both accuracy and sharpness of the cuts. Figure 4 shows the pie charts summarizing the results of the Debana Kote. Dan grade participants show higher percentage of the cuts hitting the target as compared to Kyu grade ( $92 \%$ and $75 \%$ respectively). The percentage of scoring is also significantly higher in Dan grade $(78 \%)$ than Kyu grade (37\%). The mean impact force of scoring by Dan grade (1159 \pm 379 N) was higher than Kyu grade $(852 \pm 429 \mathrm{~N}$ ) (Figure 5). T-tests performed on the force and conductivity of the cuts showed that there are significant differences in the conductivity and force of scoring between Dan grade and Kyu grade $(p<0.001)$, whereas there is no significant difference in the non-scoring cut $(p>0.3)$.
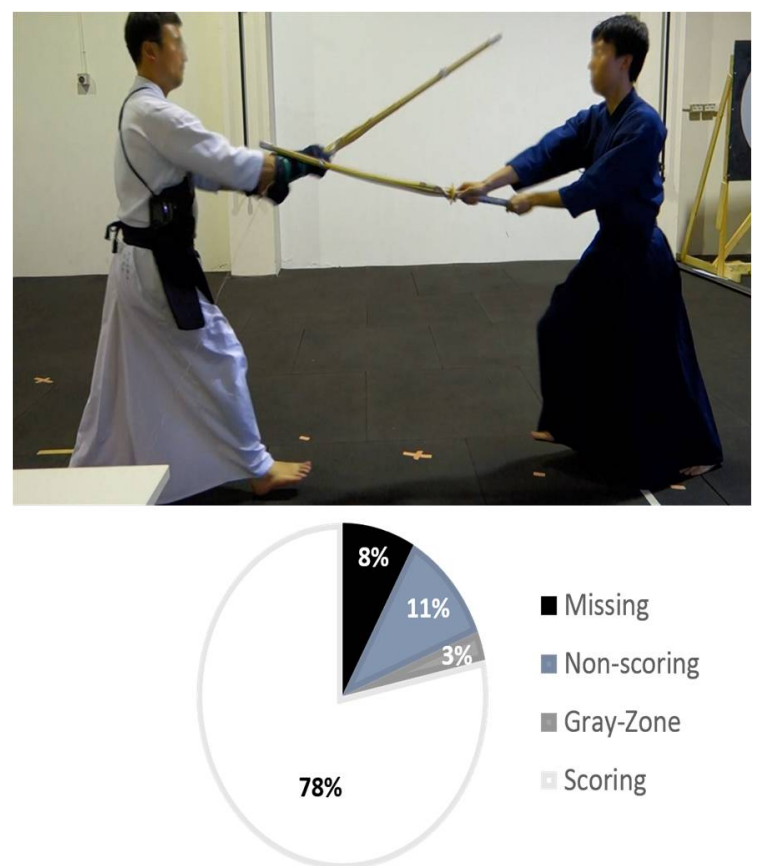

(a)
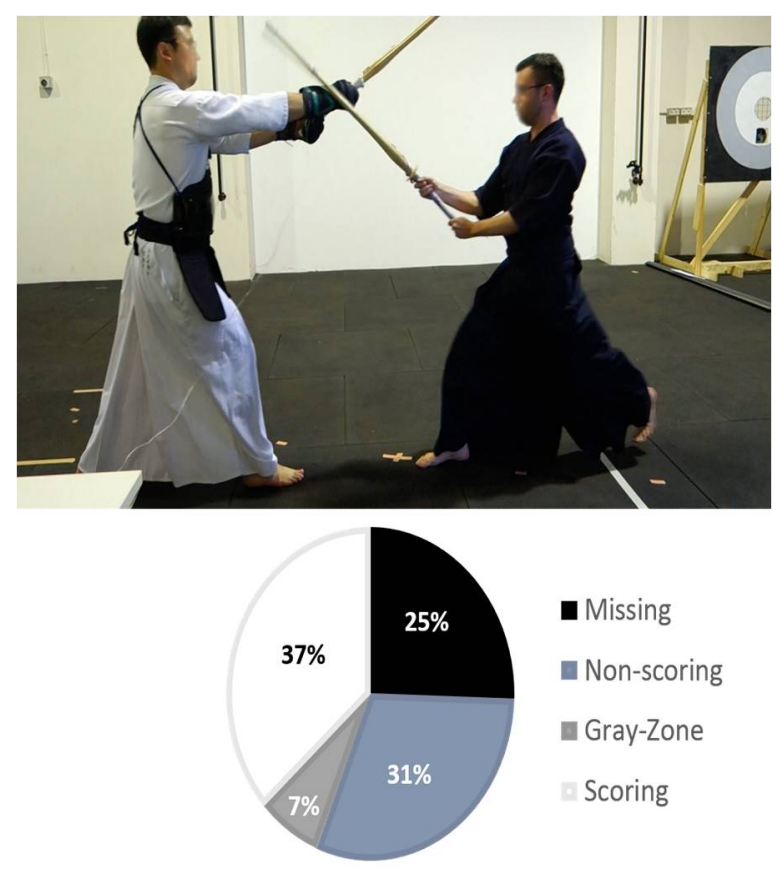

(b)

Figure 4. Photos of the Debana Kote kendo motion and pie chart summarising the percentage of scoring, grey-zone, non-scoring and missing the target in (a) Dan grade; (b) Kyu grade. 


\section{Average impact force of scoring}

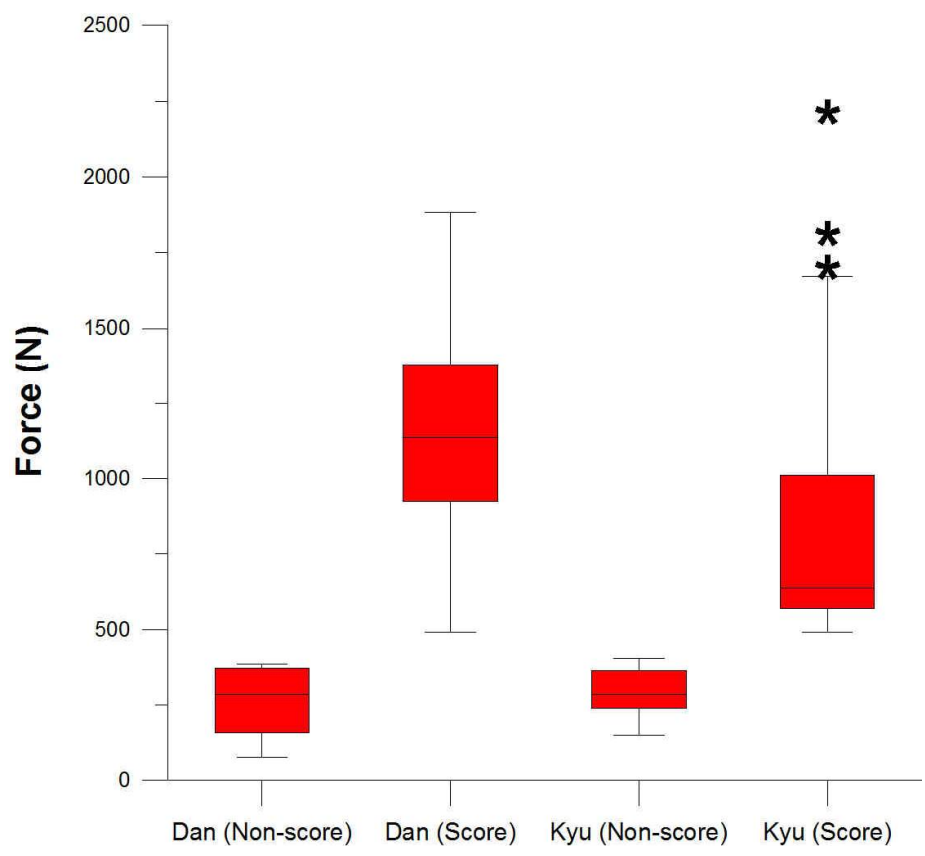

Figure 5. The box-whisker plots of average of impact force scoring and non-scoring of force $(\mathrm{N})$ in Dan grade and Kyu grade ${ }^{*}=$ outliers $)$.

\section{Discussion}

These results can be explained by the observation of the motions of the Debana Kote cut performed by Dan grade and Kyu grade participants (Figure 4). It can be seen that the Dan grade participants have smaller arm and knee flexion angles compared to the Kyu graders.

A smaller arm flexion angle is desirable in Kendo motion as it increases the length of the attack and reduce the time to reach the target. In addition to this, the smaller arm flexion angle also results in the shinai landing on top of the target, increasing the contact area of the shinai on the target. Hence, this increases the chance of scoring and reduces the occurrence of missing the target or a no score.

A larger arm flexion angle results in the shinai landing on the side of the wrist target. It has less contact surface on the target and produces less impact force. Therefore, there is a higher rate of strikes missing the target as observed in Kyu grade Kendokas and higher percentage of non-scoring strikes due to weak impact forces.

One of the hypotheses of why Dan grade Kendokas have smaller arm flexion angles is that the Dan grade Kendokas exhibit a better reaction time and have acquired more efficient motion patterns than the Kyu grade ones. Hence, they are able to reach the target faster and with greater force than the Kyu grade Kendokas, resulting in better striking accuracies and higher impact forces as observed in the results obtained from the smart Kote glove.

\section{Conclusions}

A smart Kote glove has been successfully developed with an in-house pressure platform to measure the impact force of the Kote cut. The Debana Kote technique was tested using the smart glove and the results were compared between Dan and Kyu grades. Dan grades exhibited higher accuracy and force of their Kote cut compared to Kyu grades.

Acknowledgments: The authors would like to thank the University of Melbourne Kendo Club, Australia, for participating in the study, as well as the Kendokas who volunteered to perform their kendo motions for the study.

Conflicts of Interest: The authors declare no conflict of interest. 


\section{References}

1. Cowie, J.I.; Flint, J.A.; Harland, A.R. In Wireless Impact Measurement for Martial Arts (p43), Paris, 2008; Springer Paris: Paris, France, pp. 231-237.

2. Harding, J.W.; Mackintosh, C.G.; Martin, D.T.; James, D.A. Automated scoring for elite half-pipe snowboard competition: Important sporting development or techno distraction? Sports Technol. 2008, 1, 277-290.

3. FIE (IFF). Information Letter 2; International Fencing Federation: Lausanne, Switzerland, 2007; p. 1.

4. Chi, E.H. Introducing Wearable Force Sensors in Martial Arts. IEEE Pervasive Comput. 2005, 4, 47-53.

5. James, D.A.; Uroda, W.; Gibson, T. Dynamics of Swing: A Study of Classical Japanese Swordsmanship Using Accelerometers; ASTA (Australasian Sports Technology Alliance): Melbourne, Australia, 2005; pp. 343-348.

6. Jeong, K.; Fuss, F.K.; Fuernschuss, B.; Weizman, Y. Development of a smart kendo sword and assessment of grip pressure of kamai stance and kote cut. Procedia Eng. 2015, 112, 231-236.

7. FIK, I.K.F. The Regulations of Kendo Shiai and Shinpan; Revised Edition of 2006 ed.; International Kendo Federation: Tokyo, Japan, 2006.

8. Salmon, G. Kendo: A Comprehensive Guide to Japanese Swordsmanship; Tuttle Publishing: Clarendon, NY, USA, 2013.

9. AKR. Time in Grade Policy; Australian Kendo Renmei: Melbourne, Australia, 2008.

10. Jeong, K.; Wang, X.; Fuss, F.K. Measurement of perception of impact force in kendo. 2017, submitted.

(C) 2018 by the authors; Licensee MDPI, Basel, Switzerland. This article is an open access article distributed under the terms and conditions of the Creative Commons Attribution (CC BY) license (http://creativecommons.org/licenses/by/4.0/). 